\title{
Anatomical Features and Clinical Importance of the Vertebral Artery
}

Ace Dodevski, Dobrila Tosovska-Lazarova

Institute of Anatomy, Faculty of Medicine, University Ss Cyril and Methodius Skopje, Skopje, Republic of Macedonia

\footnotetext{
Citation: Dodevski A, Tosovska-Lazarova D. Anatomical Features and Clinical Importance of the Vertebral Artery. Maced J Med Sci. 2012 Oct 15: 5(3):328-335, http://dx doi.org/10.3889/ MJMS.1857-5773.2012.0251.

Key words: vertebral artery; anatomy; origin; tortuosity; transverse foramen.

Correspondence: Ace Dodevski, MD. Institute of Anatomy, Medical Faculty, "Ss. Cyril and Methodius University", 50 Divizija, bb 1000 Skopje, Repubic of Macedonia Tel/fax: ++389 23125 304. E-mail: a.dodevski@medf.ukim.edu.mk

Received: 04-May-2012; Revised: 04-Jul-2012; Accepted: 04-Jul-2012; Online first: 27-Sep-2012

Copyright: (c) 2012 Dodevski A. This is an openaccess article distributed under the terms of the Creative Commons Attribution License, which permits unrestricted use, distribution, and reproduction in any medium, provided the original author and source are credited.

Competing Interests: The author have declared that no competing interests exist.
}

\section{Abstract}

The vertebral artery and its branches are target of arteriographic investigations, ultrasound and Doppler visualization, MRI and CT imaging in many contemporary diagnostic procedures. The big horizon of procedures in the interventional and diagnostic radiology, orthopedic and surgery opens new avenues for the study of anatomy and especially variations of the vertebral artery and its branches. The aim of the present report is to give a summary of the vertebral artery anatomy as well as its variations, and to emphasize there clinical importance.

\section{Introduction}

Because of their anatomic location and inconvenient access for surgical procedures, vertebral artery (VA) stayed neglected in research for a long time. Since Egas Moniz performed the first vertebral angiography in 1933, the VA has become more and more important in different clinical fields. Crawford and DeBakey described one of the first surgical approaches to this region in 1958 for lesions causing ostial stenosis. In addition to angiography, continuous wave Doppler sonography, and color coded Doppler sonography have been the most important tools for noninvasive investigation of the extra cranial part of VA for many years [1].

The brain represents just $2 \%$ of the body mass but receives about $15 \%$ of the blood minute volume. The 
brain blood supply is provided by the anterior bran circulation or carotid system and posterior brain circulation or vertebral system, which is responsible for about $30 \%$ of the brain blood supply [2].

The VA is the first and the largest branch of the subclavian artery arising from the posterosuperior aspect of its first part. It runs upwards and backward in the scalenovertebral triangle formed by muscles scalenus anterior and muscles longus colli. The common carotid artery and the vertebral vein are in front of it. It is crossed by the inferior thyroid artery and by the thoracic duct on the left side and the right lymphatic duct on the right side. The seventh cervical transverse process, the inferior cervical ganglion and ventral rami of the seventh and eight cervical spinal nerves lie posterior to the artery. Then the vessel reaches the sixth cervical vertebra to enter into the foramen of transverse processus. It passes through the foramina in the transverse processes of all of the cervical vertebrae except the seventh, curves medially behind the lateral mass of the atlas and enters the cranium via the foramen magnum. At the lower pontine border unites with its contralateral fellow to form the basilar artery [2-4].

The VA is divided into four segments. The segment of the artery from its origin at subclavian artery to its entry into the respective transverse foramen is called the pretransverse, prevertebral or V1 segment of the VA. The second segment, vertebral or V2 segment extends from the foramen transversarium (FT) of the sixth cervical vertebra till the VA exits the axis. The third segment, suboccipital or V3 segment extends from the FT of the second cervical vertebra to the foramen magnum. The fourth segment, cranial or V4 segment is intracranial and terminates as a basilar artery [1-4].

The VA supplies with blood the upper part of spinal cord and its membranes, the brain stem, cerebellum, occipital and temporal lobe of the cerebrum, muscles of the neck and inner ear [1-6].

Classical studies with anatomical dissection indicate the presence of variations of the VA. The purpose of this study is by a survey on the available literature to show the VA anatomy and variations in one summary report, and to emphasize there clinical importance.

\section{Discussion}

In anatomy, surgery, angiography and in all non-invasive procedures it is very important to know the exact course of the artery and the possible variations [1].

\section{Origin of the VA}

Although the VA is classically described as the first branch from the subclavian artery, multiple variations in the origin of this vessel have been reported in the literature [7]. Several researches have reported anomalous origin, in which origin point of the VA is from the aortic arch [1, 8-15], from the thyrocervical trunk [1, $14,16]$, from the brachiocephalic trunk [6], from the common carotid artery $[1,14,17]$, and from the external carotid artery $[1,12,14]$. Though the overall incidence of the anomalous origin of the VA is low, it occurs mostly on one side, usually on the left $[11,12]$. Anomalous origin of the right side is a rare anatomic variant [18]. The VA may have duplicate origin, generally from the aortic arch and subclavian artery [19]. Bilateral aortic arch origin of the VA is exceptional anatomic variant [7].

The complex embryologic evolution of the vessel accounts for the wide range of possible origins. The VA is formed between the $32^{\text {nd }}$ and the $40^{\text {th }}$ gestational day (7-18 mm embryo) from fusion of secondary persistent segments of cervical arteries and the primitive dorsal aortic arch. Abnormal arrangements of this fusion process undoubtedly account for any abnormal origins. It is possible that abnormalities in fusion also contribute to some tortuosities of the vessel [1].

Anomalous origin of the VA is not very common. In fact, most types have been published in a few case reports. The most common anomalous origin is left VA arising from the arch of aorta (Fig. 1). An abnormal origin

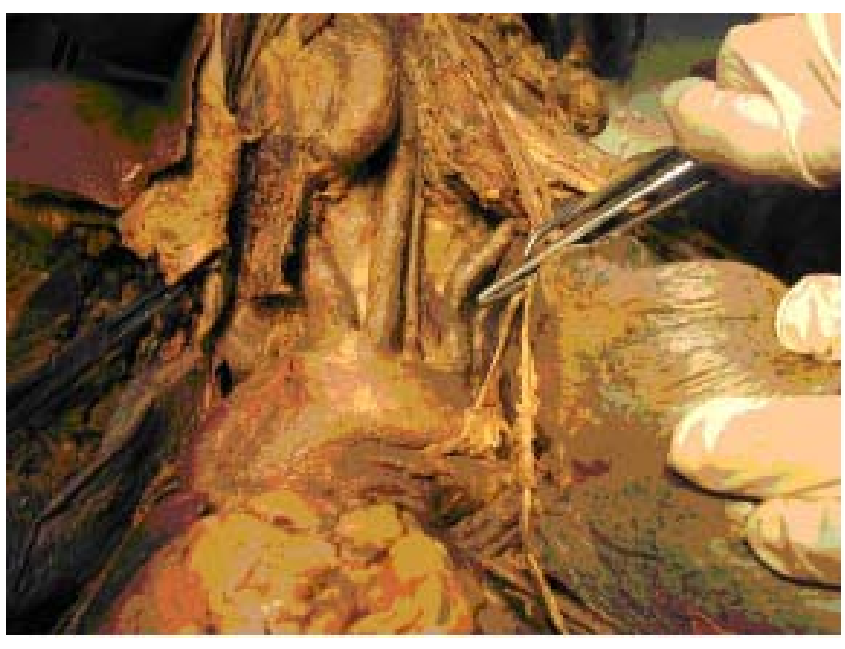

Figure 1: Variant origin of left vertebral artery from the aortic arch (published by) Imre N, Yalcin B, Ozan H. Unusual origin of the left vertebral artery. IJAV. 2010; 3: 80-2 [8]. 
of the left VA, from the aortic arch was found by Komiyama in 2.4\%; Matula 3.48\%; Zhivadinovik 3.64\%; Panicker 5\%; Mori 6.9\% [1, 11, 15, 20].

According to Matula the variations of the origin of the VA can be divided by two criteria: first, the vessel of origin of the VA and second, the origin from the subclavian artery with respect to circumferential division. Origin from a vessel other than the subclavian was found in $8(3.48 \%)$ cases. The location of the origin on the circumference of the subclavian artery was found to be cranial in 47\% (33 cases), dorsal in 44\% (31 cases), caudal in 6\% (four cases), and ventral in 3\% (two cases) [1].

Understanding the great vessels of the aortic arch and their variations are important for both the endovascular interventionist and the diagnostic radiologist. This has become more important in the era of carotid artery stents, VA stents, and new therapeutic options for intracranial interventions [13]. Anomalous VA origins also represent a potential pitfall at diagnostic cerebrovascular imaging [7]. If the VA are not identified in their normal position, this finding can be misinterpreted as the vessels being congenitally absent, or may be wrongly assumed to be occluded or diseased [7, 13]. Finally, knowledge of potential VA origin variants appears to be mandatory for cardiothoracic surgical planning or endovascular interventions [7, 13]. The true value of detecting anomalous origins is the diagnostic gain prior to the surgery of supraaortic arteries. For cases in which the VA originates from the carotid artery or its branches, the ligation of the common carotid artery may cause a compromise of the posterior fossa blood supply [18].

In most cases described in the literature, anomalous VA origin was not presented with clinical symptoms [7, 13, 18]. In rare cases, patients complained of dizziness and vertigo, which was thought to have no connections to the anomalous origin of the VA $[13,18]$. Bernardi and Dettori in their study hold the hypothesis that anomalies of origin, of caliber and of distribution of the large vessels of the aortic arch may favor cerebral disorders because of alterations in the cerebral hemodynamics [21]. Anomalous origins may lead to altered hemodynamics and predispose the patient to intracranial aneurysm formation. Therefore, in patients with these anomalies, a thorough search for coexisting aneurysms should be undertaken. Endovascular therapy of intracranial aneurysms can be performed before they present clinically as subarachnoid hemorrhages or mass effect and, thereby, decrease morbidity and mortality [13]. There is no conclusive evidence that these variants lead to a predisposition to cerebrovascular disorders [22].

The VA dissection is a potentially disabling and yet probably under recognized condition often occurring in young and middle-aged adults. The mean age of symptom onset is about 40 years. But VA dissection can occur also in children and in patients older than 60 years [23].

Komiyama et al. in their study examined the incidence of VA dissection and its relationship to the origin of the VA from either the aorta or subclavian artery. Arterial dissection of the VA was detected in 17 patients, an incidence of $1.9 \%$. According to their studies left VA of aortic origin was associated with a significantly higher incidence of arterial dissection of its own vessel than left VA of left subclavian artery origin $(p<0.001)$ and right VA of right subclavian origin $(p<0.001)$. The reasons for the high incidence of arterial dissection associated with VA of aortic origin remain to be elucidated. However, there could be two anatomical explanations: congenital structural defects of arterial wall and alteration of cerebral hemodynamics [20].

According to the anatomic location of VA dissection different studies reported conflicting results. In the study conducted by Arnold et al. VA dissection was more often in the second and third segment of the artery than in the prevertebral or intracranial segment of the artery [23]. On the other hand Shin et al. reported that the most frequent site of VA dissection was the intracranial segment, followed by second, third and the first segment of the artery [24]. Other authors such Provenzale and Pelkonen didn't found preferred site of dissection along the course of the VA in their studies $[25,26]$.

\section{Tortuosity}

From the point of origin toward foramen of transverse processus of the vertebrae, VA may show different level of tortuosity (Fig. 2). A suggested congenital origin is difficult to assess since tortuosity has been shown to increase with age [14].

Tortuosity of a VA used to be considered a rare abnormality, but recently its frequency has increased [23]. The left VA is involved more often than the right one [24]. This may be related to the fact that the left VA is larger than the right in a higher percentage of individuals [25]. Rarely, bilateral or multilevel tortuosity of the VA occurs with corresponding radiologic findings [26].

Matula et al. in their series found that in $61 \%$ of 


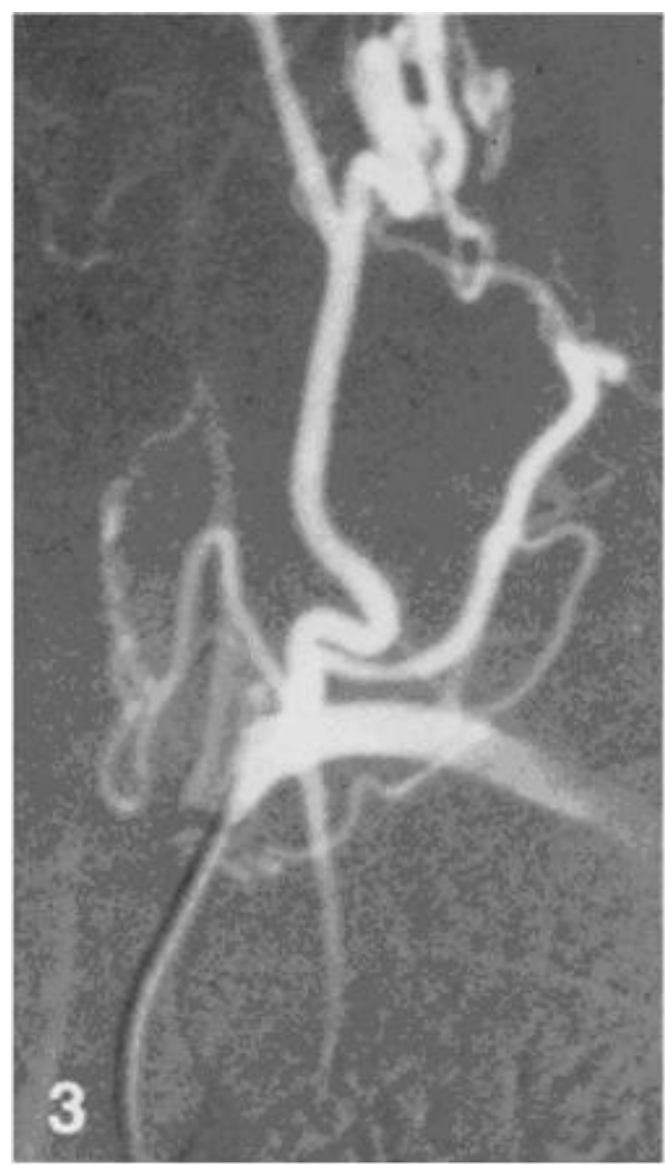

Figure 2: Frontal tortuosity of the vertebral artery (published by) Tratting S, Matula C, Karnel F, et al. Difficulties in examination of the origin of the vertebral artery by duplex and colour-coded Doppler sonography: anatomical considerations. Neuroradiology. 1993; 35:2969 [14].

the cases the VA followed non-tortuous path from the origin to the transverse foramen and in 39\% followed a tortuous path. Furthermore, they classified tortuosity according to the geometric plane as horizontal (44.9\%), sagittal (33.7\%), and frontal (21.4\%). In $32.5 \%$ of the cases the contorted pathway was on the right side, and in $68 \%$ of the cases, on the left [1].

Trattnig et al. in their series found that $52.8 \%$ of the examined vessels, followed a relatively straight course from their origin to their entry into the transverse foramen, but $47.2 \%$ showed some form of tortuosity (one coil at most). The main plane of the tortuosity was transverse in $42.5 \%$, sagittal in 30\% and frontal in $27.5 \%$ [14].

Poonam et al. in their series found that a total of 31 VA (22.1\%) showed the tortuous pathway in 25 cadavers. Bilateral tortuosity was encountered in six cadavers and unilateral tortuosity in 19 cadavers (13 on the left and 6 on the right). Twenty-six VA had a single coil tortuosity and five VA had a double coil tortuosity. The maximum number of tortuosities was observed in the transverse plane, followed by tortuosity in the sagittal plane [31].

Sastry et al. observed tortuous course in nine cases or $23.7 \%$ and in all cases the plane of the tortuosity was horizontal [32].

Clinically, tortuosity of the VA does not have a hemodynamically significant consequence [1]. The presence of a tortuous VA may go undetected because of the lack of symptoms. However, loops of the vessel have been described to cause several problems. Loops of the VA have been reported to cause radicular symptoms via nerve root compression $[1,14]$. The VA loop formation is one of those infrequent causes which can cause pressure erosion of the adjacent vertebral body, widening of the intervertebral foramen and compress the cervical nerve root causing cervical radiculopathy [33]. Various symptoms can occur depending on the level of the anomaly. If the anomalies are at higher levels of the VA, the clinical presentation will be of dysphagia, glossopharyngeal neuralgia, Horner's syndrome, occipital neuralgia and spasmodic torticolis [33]. The anomalies at the lower levels can cause symptoms of cervicobrachial neuralgia [27, 28, $29,30,33$ ]. Cervicobrachial neuralgia produced by vascular compression presents with paraesthesia and dysaesthesia of the fingers without a triggering factor, the lack of nocturnal symptoms, and the rarity of neurologic deficits [33]. Symptomatic loops may be corrected by a bypass procedure $[1,14]$.

Cervical spinal fracture has been reported secondary to bony erosion from a VA loop in contact [1]. Also, a vertebral loop caused by displacement from a mass lesion in the scalenovertebral trigone may be compressed and lead to vertebrobasilar insufficiency [33]. Angiographic or sonographic description of VA tortuosity in patients with signs and symptoms of vertebrobasilar insufficiency has led to surgical correction $[1,14]$.

When a tortuous VA is imaged, clinical correlation is essential to determine whether the abnormality is responsible for the patient's signs and symptoms. If a cause and effect are not clear, conservative treatment is preferred. However, symptoms have been relieved by surgical intervention in some cases when radiculopathy was caused by nerve root compression due to vascular 
anomaly [27].

The anterior approach for the decompression of the cervical spinal cord and nerve roots is an accepted surgical treatment for cervical radiculopathy. Although the reported results are generally excellent, the procedure has some potential complications, such as VA laceration [34].

In the presence of an abnormally tortuous VA, using anatomic landmarks to guide decompression may not prevent iatrogenic injury to the VA. Failure to recognize this anomaly during preoperative planning can lead to laceration of the vessel, even when decompression is achieved within generally accepted safe limits of lateral decompression [35].

From a surgical standpoint it can be of great importance to know whether the VA is straight or tortuous in, for example, surgical transposition of the VA to the carotid artery because of high-grade stenosis at its origin $[1,14]$.

\section{The level of entry into the FT}

The most important variation is the level of entrance of the VA into the transverse foramen (Fig. 3). Most commonly the artery enters the transverse foramen of sixth cervical vertebra. But in variable percentage of cases, the VA enters the $\mathrm{C} 7, \mathrm{C} 5, \mathrm{C} 4$, or even the $\mathrm{C} 3$ and C2 transverse foramen. In case of entrance above C6, the VA runs anteriorly to the C6 transverse process, between the longus colli muscle and the anterior branch of the $\mathrm{C} 6$ transverse process. The VA is thus unprotected by bony structures and at risk of injury when the muscle is divided. Thus, recognizing this anatomic variation on preoperative investigations is important to avoid any inadvertent damage [4].

Until now, several studies reporting the incidence of variable level of entrance into the transverse foramen have been published in the literature. According to the available data from these studies Matula et al. found level of entry of the VA into the transverse foramen at C6 in $91 \%$ of their cases. They also found two cases of the VA entering at C4 and C5 and two cases of VA entering at the level of $\mathrm{C} 7[1]$.

In the study conducted by Kajimoto et al. 37 of 40 dissected VA entered the transverse foramen at the sixth cervical vertebra (C6-92.5\%) and three (7.5\%) of them through $\mathrm{C} 7$ transverse foramen [36].

Bruneau et al. found that in $93 \%$ of all specimens

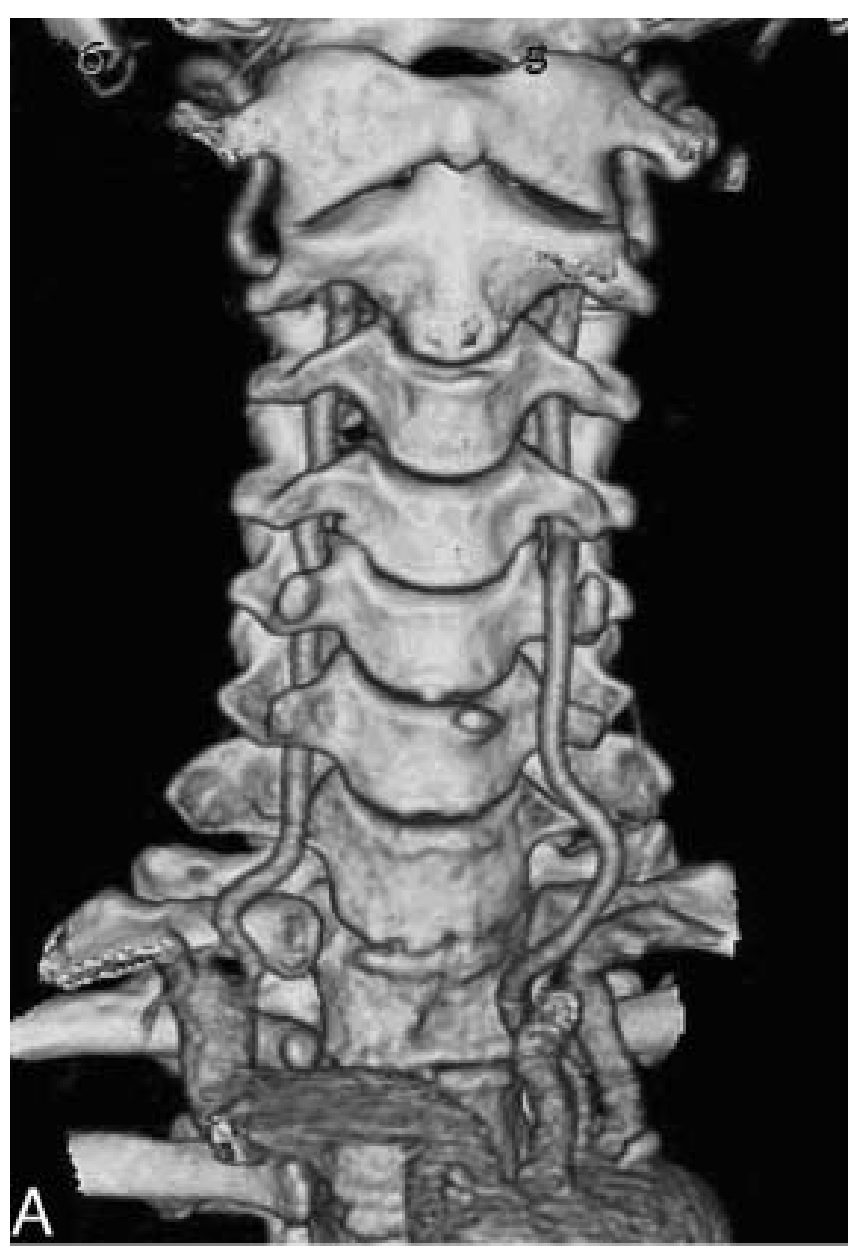

Figure 3: Three-dimensional CT angiography showing abnormal entrance of the VA into the left C4 transverse foramen (published by) Hong JT, Park DK, Lee MJ, Kim SW, An HS. Anatomical variations of the vertebral artery segment in the lower cervical spine Spine. 2008;33(22):2422-6 [37].

VA entered the transverse foramen of C6 vertebrae. An abnormal level of entrance was observed in $7 \%$ of the specimens, with a level of entrance into the $\mathrm{C} 3, \mathrm{C} 4, \mathrm{C} 5$, or C7 transverse foramen, respectively, in $0.2 \%, 1 \%, 5 \%$ and $0.8 \%$. Seventeen abnormalities were right-sided and eighteen were left-sided. Thirty one out of 250 patients had a unilateral anomaly and two had a bilateral anomaly [4].

Hong et al. in their study found that the VA entered the C6 transverse process in $94.9 \%$ of the specimens. Abnormal VA entrance was observed in $5.1 \%$ of the specimens, with entrance into the $\mathrm{C} 4, \mathrm{C} 5$, or C7 transverse foramen in $1.6 \%, 3.3 \%$, and $0.3 \%$, respectively [37].

There is a correlation between the artery entering 
into the foramen at an unusual level and a variation in its origin from the subclavian artery. Based on the findings of Yamaki et al. the right and left VA that arose from the subclavian arteries had the most common entrance at the transverse foramen of C6. The right and left VA that arose from other than the subclavian artery had a high frequency of entrance at C3, C4 or C5 [38].

It is important to know anatomic variations of the VA and to look for them systematically on preoperative investigations to avoid inadvertent tearing during anterior or lateral approach to the cervical spine. These anatomic variations of the entrance level can be detected easily on MRI and contrast enhanced CT scans. These variations could be missed on a noninjected CT scan, but should be suspected nevertheless if a transverse foramen appears to be small. When facing a small transverse foramen, the differential diagnoses are a hypoplastic or aplastic VA or an unfilled foramen because of the upper level of entrance. In such a case, MRI or CT scan is mandatory if surgery is performed in the VA vicinity [39].

The indications for surgery in the vicinity of the VA include spondylosis, degenerative disc disease, benign and malignant bone tumors, infection, and trauma. With each of these surgical indications, there is the potential for damage to the VA during the extent of the decompression. Thus, an understanding of the anatomy of $\mathrm{VA}$ and the surrounding bony anatomy is essential to prevent iatrogenic VA injuries [35]. Iatrogenic VA injuries can occur from posterior cervical surgery in the spine; however, these injuries are rare [35].

The true incidence of VA injury during anterior cervical spine surgery is unknown because of underreporting of such occurrences [40]. Considering unrecognized and underreported cases, the true incidence of VA injury will probably remain unknown and underestimated, and will increase with the increasing popularity of cervical instrumentation techniques [41].

The incidence of VA injury during anterior cervical procedures based on retrospective analysis was reported in $0.3 \%$ of the patient by Golfinos, Burke found also $0.3 \%$, and $0.5 \%$ by Smith et al [42-44].

Consequences of VA injury are often unpredictable, with a wide spectrum of symptoms. Although some patients may remain asymptomatic due to adequate collateral circulation, other patients may sustain devastating vertebrobasilar ischemia or fatal bleeding [41].

Laceration of the VA is the most challenging of surgical dilemmas during anterior cervical spine surgery, as ganging control of the massive hemorrhage from a ruptured VA is difficult and could possibly result in an uncertain neurologic morbidity. The VA comprise the posterior circulation of the brain, which includes the occipital lobes, the brain stem, and the labyrinthine branches to the inner ear. Therefore, even though the bleeding from the artery can be controlled, ischemic neurologic consequences may occur in some individuals. The potentially devastating complications of VA injury with its supply of the cerebellum, brainstem and spinal cord are well known: brain stem infarction or central respiratory dysfunction [45].

When a VA laceration occurs, there is disagreement as to the optimal method to manage this potentially devastating complication. The various options available include direct ligation, use of thrombostatic agents, endovascular techniques, etc. After vascular control of a VA laceration, it is mandatory to obtain radiographic imaging confirmation of the adequacy of the repair or ligation. CT angiography or MR angiography is necessary to exclude a growing pseudoaneurysm [35].

There is no doubt that prevention of the problem is the best treatment. Careful preoperative planning is essential in cases with unusual anatomy of the VA and helps avoiding potentially life threatening complications $[35,37,41,42,45]$.

\section{Conclusion}

In conclusion, based on the review of the literature we can conclude that the anatomic variations of the VAare common. Although anatomically interesting, an awareness of the VA anatomy and variations is clinically important. This knowledge find clinical application in diagnostic investigation and interpretation of the pathology of the VA such as CT, MRI, angiographic and ultrasound investigations and treatment of VA pathology during surgical and endovascular procedures.

\section{Acknowledgements}

Publication fee for this review article was covered by Ss Cyril and Methodius University Skopje PhD School. 


\section{References}

1. Matula C, Tratting S, Tschabitscher M, Day JD, Koos WT. The course of the prevertebral segment of the vertebral artery: Anatomy and clinical significance. Surg Neurol. 1997;48:12531.

2. Papazova M, Lazarova D, Zhivadinovic J. Vaskularizacija na mozokot. 1 ed. Skopje: UKIM; 2010.

3. John D. Langdon. Neck. In: Gray's H. Anatomy. The Anatomical Basis of Clinical Practice. 39 ed. New York: Elsevier, 2005:531-65.

4. Marinkovic S, Milisavljevic M. Arteria vertebralis. In: Marinkovic S, Milisavljevic M, Antunovic V. Arterije mozga i kicmene mozdine: Anatomske i klinicke karakteristike. 1ed. Beograd: Bit inzenjering, 2001:30-9.

5. Bruneau M, Cornelius JF, George B. Anterolateral approach to the $\mathrm{V} 1$ segment of the vertebral artery. Neurosurgery. 2006; 58: 215-9.

6. Bhatia K, Ghabriel MN, Henneberg M. Anatomical variations in the branches of the human aortic arch: a recent study of a South Australian population. Folia Morphol. 2005; 64 (3): $217-$ 24.

7. Albayram S, Gailloud P, Wasserman BA. Bilateral arch origin of the vertebral arteries. AJNR Am J Neuroradiol. 2002; 23:4558.

8. Imre N, Yalcin B, Ozan H. Unusual origin of the left vertebral artery. IJAV. 2010; 3: 80-2.

9. Kubikova E, Osvaldova M, Mizerakova P, Faloudy $H$, Benuska J. A variable origin of the vertebral artery. Bratisl Lek Listy. 2008; 109 (1):28-30.

10. Nayak SR, Pai MM, Prabhu LV, Sujatha D, Prakash S. Anatomical organization of aortic arch variations in the India: embryological basis and review. J Vasc Bras. 2006; 5 (2): 95100.

11. Panicker HK, Tarnekar A, Dhawane V, Ghosh SK. Anomalous origin of left vertebral artery - embryological basis and applied aspects - a case report. J Anat Soc India. 2002; 51 (2): 234-5.

12. Poonam SRK, Sharma T. Incidence of anomalous origins of vertebral artery-Anatomical study and clinical significance. Journal of Clinical and Diagnostic Research. 2010; 4:262631.

13. Satti SR, Cernigilia A, Koenigsberg RA. Cervical vertebral artery variations: an anatomic study. AJNR Am J Neuroradiol. 2007; 28:976-80.

14. Tratting S, Matula C, Karnel F, et al. Difficulties in examination of the origin of the vertebral artery by duplex and colour-coded Doppler sonography: anatomical considerations. Neuroradiology. 1993; 35:296-9.
15. Zhivadinovik J, Matveeva N, Jovevska S. Anatomic variation in the origin of aortic arch branches. Acta Morpholo. 2009; 6 (2): 15-8.

16. Strub WM, Leach JL, Tomsick TA. Left vertebral artery origin from the thyrocervical trunk: A unique vascular variant. AJNR Am J Neuroradiol. 2006; 27: 1155-6.

17. Buckenham TM, Wright IA. Ultrasound of the extracranial vertebral artery. Brit J Radiol. 2004; 77: 15-20.

18. Lemke AJ, Benndorf G, Liebig T, Felix R. Anomalous origin of the right vertebral artery: review of the literature and case report of the right vertebral artery origin distal to the left subclavian artery. AJNR Am J Neuroradiol. 1999; 20: 131821.

19. Komiyama M, Nakajima H, Yamanaka K, Iwai Y. Dual origin of the vertebral artery. Neurol Med Chir. 1999; 39: 932-7.

20. Komiyama M, Morikawa T, Nakajima H, Nishikawa M, Yasui T. High incidance of arterial dissection associated with left vertebral artery of aortic origin. Neurol Med Chir. 2001; 41:812.

21. Bernardi L, Dettori P. Angiographic study of a rare anomalous origin of the vertebral artery. Neuroradiology. 1975; 9:43- 7 .

22. Wasserman BA, Mikulis DJ, Manzione JV. Origin of the right vertebral artery from the left side of the aortic arch proximal to the origin of the left subclavian artery. AJNR Am J Neuroradiol. 1992;13:355-8.

23. Arnold M, Bousser MG, Fahrni G, et al. Vertebral artery dissection: presenting findings and predictors of outcome. Stroke. 2006;37:2499-503.

24. Shin JH, Suh DC, Choi CG, Lee HK. Vertebral artery dissection: spectrum of imaging findings with emphasis on angiography and correlation with clinical presentation. Radio Graphics. 2000;20:1687-96.

25. Provenzale JM, Morgenlander JC, Gress D. Spontaneous vertebral dissection: clinical, conventional angiographic, CT, and MR findings. J Comput Assist Tomogr. 1996;20:185-93.

26. Pelkonen O, Tikkakoski T, Leinonen S, et al. Extracranial internal carotid and vertebral artery dissections: angiographic spectrum, course and prognosis. Neuroradiology. 2003;45:717.

27. Kricun R, Lawrence PL, Winn RH. Tortuous vertebral artery shown by MR and CT. AJNR Am J Neuroradiol. 1992;159:6135.

28. Lindsey RW, Piepmeier J, Burkus JK. Tortuosity of the vertebral artery: an adventitious finding after cervical trauma. A case report. J Bone Joint Surg Am. 1985;67:806-8.

29. Ono SE, Kawasaki CS, Coelho LOM, Lucaski FF, Neto AC. Widening of intervertebral foramen by tortuous vertebral 
artery. Arq Neuropsiquiatr. 2009;67(1):115-6.

30. Zimmerman HB, Farreli WJ. Cervical vertebral erosion caused by vertebral artery tortuosity. Am J Roentgenol. 1970;108(4):767-70.

31. Poonam RKS, Rathore NJ. Tortuous vertebral arteriesincidence and clinical implications. J Clin Diagnostic Res. 2011;5(4):780-2.

32. Ranganatha Sastry V, Manjunath KY. The course of the V1 segment of the vertebral artery. Ann Indian Acad Neurol. 2006;9:223-6.

33. Hoon SK, June HL, Gene C, Lee SH. Cervical radiculopathy caused by vertebral artery loop formation: a case report and review of the literature. J Korean Neurosurg. 2010;48:465-8.

34. Oga M, Yuge I, Terada K, Shimizu A, Sugioka Y. Tortuosity of the vertebral artery in patients with cervical spondylotic myelopathy: risk factor for the vertebral artery injury during anterior cervical decompression. Spine. 1996; 21(9):1085-9.

35. Heary RF, Albert TJ, Ludwig SC, et al. Surgical anatomy of the vertebral arteries. Spine. 1996;21:2074-80.

36. Kajimoto BHJ, Addeo RLD, Campos DC, et al. Anatomical study of the vertebral artery path in human lower cervical spine. Acta Ortop Bras. 2007;15(2):84-6.

37. Hong JT, Park DK, Lee MJ, Kim SW, An HS. Anatomical variations of the vertebral artery segment in the lower cervical spine. Spine. 2008;33(22):2422-6.
38. Yamaki K, Saga T, Hirata T, et al. Anatomical study of the vertebral artery in Japanese adults. Anatomical Science International. 2006;81:100-6.

39. Bruneau M, Cornelius JF, Marneffe V, Triffaux M, George B. Anatomical variations of the V2 segment of the vertebral artery. Neurosurgery. 2006;59(1):20-4.

40. Pait TG, Killefer JA, Arnautovic KI. Surgical anatomy of the anterior cervical spine: the disc space, vertebral artery and associated bony structures. Neurosurgery. 1996;39 (4):76976.

41. Nam KH, Sung JK, Park J, Cho DC. End-to end anastomosis of an unanticipated vertebral artery injury during C2 pedicle screwing. J Korean Neurosurg Soc. 2010;48:3636.

42. Smith MD, Emery SE, Dudley A, Murray KJ, Leventhal M. Vertebral artery injury during anterior decompression of the cervical spine. J Bone Joint Surg. 1993;75(3):410-5.

43. Burke JP, Gerszten PC, Welch WC. Iatrogenic vertebral artery injury during anterior cervical spine surgery. Spine. 2005;508-14.

44. Golfinos JG, Dickman CA, Zabramski JM, Sonntag VK, Spetzler RF. Repair of vertebral artery injury during anterior cervical decompression. Spine.1994;19:2552-6.

45. Lu J, Ebraheim NA. The vertebral artery: surgical anatomy. Orthopedics. 1999;22 (11):1081-5. 